\title{
Warfarin prescription and administration: reducing the delay, improving the safety
}

Rebecca Dyar, Simon Hall, Bethannie McIntyre

University Hospitals Bristol Foundation Trust

\begin{abstract}
Warfarin is frequently administered to hospital patients. The prescription and administration of this medication are particularly susceptible to error. Factors contributing to this include the narrow therapeutic index, patient-specific target range, and the need for regular INR monitoring NICE guidelines state that warfarin should be given at the same time every day and the Bristol Royal Infirmary guidelines are warfarin to be given at 14:00. The 14:00 dosing ensures standardisation of administration; poor adherence to this recommendation may cause patient harm. We noticed that many warfarin doses were often given outside of maximal staffing hours and it was often left to the on call doctor to prescribe warfarin at erratic and inconsistent times.
\end{abstract}

Our primary aim was to reduce the number of adverse outcomes associated with warfarin prescription and administration. We targeted two system measures: the proportion of warfarin administrations occurring within an hour of the 14:00 prescription and the proportion of INR results outside target range. We employed the model for improvement and carried out our project across seven acute medical wards. Baseline data showed that only $24 \%$ of doses were being given within an hour of the recommended time and $64 \%$ of doses were being given after 17:00 during minimal staffing hours. We successfully introduced a warfarin box within our trust which demonstrated an improvement in warfarin administration from $24 \%$ of patients receiving their warfarin within an hour of $14: 00$ to $49 \%$ and this was subsequently associated with a reduction in INRs above target range (23\% to $9 \%)$.

\section{Problem}

Warfarin is a commonly prescribed medication and continues to be an important therapy in the prevention of arterial and venous thrombosis, and furthermore in the prevention of stroke related to atrial fibrillation[1-4]. Due to the therapeutic rise in international normalised ratio (INR), the predominant risk of warfarin is bleeding, which has the potential to cause significant morbidity or mortality if not monitored closely[5]. However, for many patients the increased risk of clot formation or stroke means that the risk of harm from warfarin therapy is acceptable and outweighed by the benefits.

The guidance for warfarin administration at University Hospitals Bristol has changed from 18:00 to $14: 00$ daily[6]. Warfarin is a "critical" medication, meaning that each dose should be administered within 60 minutes of the prescribed time on the drug chart.

Our project aimed to improve the prescription and administration of warfarin so that we were meeting our trust guideline of 14:00. Firstly, we aimed to increase the proportion of warfarin doses administered correctly as a process measure (ie within 60 minutes of the prescription time) and secondly, as an outcome measure, to reduce the number of "INR over 5" incidents occurring monthly.

Frequently, patients do not receive their warfarin within 60 minutes of the time prescribed. This introduces potential safety issues:

- Reducing or increasing the interval between doses may result in a less stable INR pattern
- A less stable INR pattern may increase the likelihood for INRs to move outside of the target range

- If warfarin is not prescribed before $5 \mathrm{pm}$, the on-call doctor will be called to write the prescription: involving a busy doctor who will likely be less familiar with the patient than their regular doctor. This increases the risk of prescription errors, inaccurate dosing.

\section{Background}

Warfarin is the most commonly used oral anticoagulant and it is a frequently prescribed medication in the hospital setting. Given its narrow therapeutic index and need for regular INR monitoring it is often susceptible to medication incidents. NICE guidelines state that warfarin should be given at the same time every day and the Bristol Royal Infirmary guidelines are warfarin to be given at 14:00[3,4]. Traditionally warfarin is given at $18: 00$ as per community dosing times, however our trust found the 14:00 dosing ensures standardisation of administration and ensures that doses are given at a time of maximal staffing to ensure prescriptions aren't missed. Furthermore a previous quality improvement project carried out at another trust showed that changing the time of administration from 18:00 to $14: 00$ was associated with a reduction in the number of warfarin doses given out of hours[7].

Warfarin doses are prescribed individually, requiring a doctor's signature for each one. In the hospital setting warfarin dosing may require continuous adjustment to achieve a stable INR within the target range, for example due to catabolic illness status and new medications prescribed. This means that often only one to three 


\section{BMJ Quality Improvement Reports}

doses of warfarin are prescribed at a time for in-patients.

Dosing is based on the INR result, generated by the hospital laboratory technicians from a "clotting tube" blood sample (result usually received within a couple of hours of arriving at the lab, unless sent in an "urgent" bag).

\section{Baseline measurement}

We recorded baseline data from two buildings in our hospital; four wards in the "new" hospital and three wards in the "old" hospital.

Baseline data was collected twice weekly, over a month period. We recorded the following each day for all patients taking warfarin: administration time of warfarin, the time the INR result was available on the hospital computer system (if one had been requested), and the INR result (with a reference target range for each patient). Data for patients on a warfarin loading regimen was not identified and therefore sub therapeutic INRs could not be reliably analysed.

Data was collated onto a run chart to compare times of warfarin administration each day (including a daily average). Tabulated results and the run chart are attached. Of 111 warfarin dosing events recorded, 27 (24\%) were given within an hour of 14:00. Forty (36\%) doses were administered before 17:00. Of 100 INR events reported, $23 \%$ were above patient-specific target range. $89 \%$ INR reports were available before 14:00.

See supplementary file: ds3554.pdf - "Baseline data warfarin project for BMJ"

\section{Design}

First intervention: education of prescribers and administrators

In our trust the principal prescribers are junior doctors. Prescribing staff were targeted through focused teaching sessions and posters on wards. Administration is carried out by nursing staff. Nurses were targeted through ward-based patient safety briefs and posters on wards (co-ordinated by charge nurses). Posters highlighted the following key safety issues:

1. Send INR with morning bloods

2. Doctors to dose warfarin in the morning

3. Give warfarin at $14: 00$

4. Make warfarin patients clear to the team.

Second intervention: warfarin boxes

To intervene at a system level, we introduced "warfarin boxes" (figure 1) in a prominent location on each ward. Warfarin charts were to be placed in the box following the 12:00 nursing drug round to act as a visual prompt for doctors to prescribe in advance of 14:00. Nursing staff could then retrieve all charts from one clearly identified location.

\section{Strategy}

PDSA cycle 1: educational intervention

Education about timely warfarin administration focused on prescribing staff (junior doctors) and administration staff (ward staff nurses). No improvement in administration times demonstrated, small reduction in INR reports above patient-specific target range.

PDSA cycle 2: warfarin box

Intervening at a system level, "warfarin boxes" were introduced on test wards. Improvements were seen in daily average administration times and proportion of doses given "on-time", in addition to a reduction in the proportion of INR reports above patient-specific target range.

See supplementary file: ds3556.pdf - "PDSA Cycles 1 and 2 Warfarin Ql"

\section{Post-measurement}

Baseline: 111 dose administrations recorded. Twenty-seven (24\%) were given within an hour of 14:00 prescription, and 40 (36\%) were given before 17:00. Number of INRs recorded that were above patient specific target range: $23(23 \%)$.

After first intervention: 78 dose administrations recorded. Ten (13\%) were given within an hour of 14:00 prescription. Twenty-one (23\%) were given before 17:00. Number of INRs recorded that were above patient specific target range: 19 (19\%).

After second intervention: 102 administrations recorded. Fifty (49\%) given within an hour of 14:00 prescription, and 64 (63\%) given before 17:00. Number of INRs recorded that were above patient specific target range: nine (9\%).

Please see the attachment for tabulated results and run chart showing daily and period average times of warfarin administration. Also included is figure 1 : warfarin box.

See supplementary file: ds4588.pdf - "Warfarin project upload file"

\section{Lessons and limitations}

One of our biggest problems was the failure of the first intervention to have an impact on the time of warfarin administration, although it did have an effect on reducing the variability of dosing times. The first intervention may not have worked because it failed to capture all of the relevant people involved in the process.

We educated junior doctors during junior doctor teaching sessions. However, rotas do not always allow the doctors to attend specific teaching sessions due on-call commitments, study leave or annual leave. Furthermore, our sessions with nursing staff may not have captured all nurses due to shift patterns (not co-ordinated with our education sessions). As the sessions were discrete events, they did 
not promote continuity of the project. It was our experience that education was not sustainable for this project; since doctors rotate every fourth months, the importance of warfarin timing and administration would require regular education sessions (which would be time consuming and labour intensive). Building an intervention like the warfarin box into the regular ward process meant that regular staff could promote a system that was already in place for new doctors when they arrived on the ward.

Regarding the process measure 'INRs outside patient-specific target range': we were unable to identify INRs that were erroneously below target range, meaning that we could only account for INRs erroneously above target range. This was because our data collection did not account for patients who had a subtherapeutic INR secondary to being loaded on warfarin. In this instance a patient being below target range is not necessarily a fault of warfarin administration but is considered part of the normal loading process. INRs below and above target range can cause harm to patients; an INR below target range is associated with an increased clotting risk, while an INR above target range is associated with increased bleeding risk. We therefore missed an opportunity to identify patients at a higher clotting risk due to having INRs below target range.

In some cases we found that delayed administration was due to patients' expectation to receive their warfarin dose at 18:00 (as in the community) and in some cases they even requested to have it at this time despite the nursing staff offering it at 14:00. This acted as a barrier to our intervention.

A few of our project medical wards had only small numbers of patients taking warfarin. In some cases this was due to an increase in patients being on the newer oral anticoagulants. As a result, two out of the seven wards reported difficulty in implementing the warfarin box as it was not needed on a daily basis. The infrequent need for and use of the box made it more difficult to maintain awareness of the issues associated with warfarin prescribing and administration.

Nursing staff often felt it was hard to push the issue of a warfarin prescription to a doctor who may be busy with medically unwell patients. They felt that warfarin prescription was often low on a doctor's list of priorities, especially when they were busy during the middle of the day.

\section{Conclusion}

This quality improvement project successfully targeted consistent prescription and administration of warfarin at the trust recommended time of 14:00.

The first intervention focused on education and although it did not produce an improvement in the daily mean time of administration, there was a reduction in the variability of the daily mean times (as demonstrated in the run chart).

Through introduction of a "warfarin box" we were able to improve the number of doses given within an hour of the recommended time and the daily average of administration time. There was a subsequent decrease in the number of INRs above target range which we envisage may aid in reducing the number of associated adverse events.

\section{References}

1. Reynolds MW, Fahrbach K, Hauch O, et al. Warfarin anticoagulation and outcomes in patients with atrial fibrillation: a systematic review and meta-analysis. Chest 2004; 126:1938.

2. Cundiff DK. Anticoagulation therapy for venous thromboembolism. MedGenMed 2004; 6(3):5.

3. National Institute for Health and Care Excellence. Clinical Guideline 180: Atrial fibrillation: The management of atrial fibrillation. Excellence NIfC, editor. London: NICE, 2014.

4. National Institute for Health and Care Excellence. Clinical Guideline 92: Venous thromboembolism: reducing the risk: Reducing the risk of venous thromboembolism (deep vein thrombosis and pulmonary embolism) in patients admitted to hospital. Excellence NIfC, editor. London: NICE, 2010.

5. Wysowski D, Nourjah P, Swartz L. Bleeding complications with warfarin use: a prevalent adverse effect resulting in regulatory action. Arch Intern Med $2007 \mathrm{Jul}$ 9;167(13):1414-9.

6. Price S. Oral Anticoagulation Guidelines-Adult. University Hospitals Bristol NHS Foundation Trust, 2011.

7. Warcel D, Johnson D, Shah $\mathrm{N}$ et al. Service improvement system to enhance the safety of patients admitted on longterm warfarin. BMJ Qual Improv Report 2014;3(1).

\section{Declaration of interests}

No interests to declare.

\section{Acknowledgements}

Dr Kate Burley, core medical trainee at University Hospitals Bristol NHS Foundation Trust.

Elin Wallis, lead anticoagulation pharmacist at University Hospitals Bristol NHS Foundation Trust. 